\title{
Printing
}

Katarina Itrićc ${ }^{\star}$ Damir Modrić and Marin Milković

\section{Edge spread function for the paper-ink system}

https://doi.org/10.1515/npprj-2018-3054

Received April 3, 2018; accepted June 8, 2018; previously published online August 3, 2018

\begin{abstract}
The importance of accurate identification of the edge spread function for the paper-ink system is closely related with the possibility of separating mechanical and optical dot gain. Our approach is based on the precise fit of the edge spread function, ESF. Namely, all of the research so far incorporates symmetric ESF, in contrast to the research reported so far, in which only the symmetric ESF has been considered, which is not realistic, due to the presence of dot gain. This approach, based on image analysis, allows us to identify uniquely the domain of impact of each contribution. Six different paper-ink systems are analyzed and discussed, and all of them show a satisfactory correlation with the proposed model.
\end{abstract}

Keywords: bidose function; dot gain; ESF; LSF.

\section{Introduction}

The evolution of technology has enabled the printing process to become more predictable and reliable. Nevertheless, there are still some unresolved issues that leave room for progress in respect to their identification, characterization and in the end, optimization. During the printing process, dot gain is one of the most important control variables because of the extremely negative impact on middle tone contrast and the overall reproduction. Some level of dot gain is unavoidable and present in all printing processes. Dot gain consists of two components as a result of two different contributions. Given their origin, they are called, respectively, optical and mechanical dot gain. Optical dot gain occurs as an outcome of scattering and diffusion of light inside paper itself (Rogers 1997, 1998). When the light approaches the boundary between halftone elements it is partially reflected, partially scattered from the surface, while the remaining part enters the substrate

*Corresponding author: Katarina Itrić, University of Zagreb, Faculty of Graphic Arts, Getaldićeva 2, 10000 Zagreb, Croatia, e-mail: kitric@grf.hr

Damir Modrić, University of Zagreb, Faculty of Graphic Arts, Getaldićeva 2, 10000 Zagreb, Croatia, e-mail: dmodric@grf.hr Marin Milković, University North, Trg dr. Žarka Dolinara 1, 48000, Koprivnica, Croatia, e-mail:marin.milkovic@unin.hr where it is absorbed after multiple internal reflections underneath the halftone element, resulting in darker and bigger halftone elements than expected. It is important to be aware of the dependency of optical dot gain on the wavelength of applied light. One possible method of modelling optical dot gain is by applying the point spread function, PSF. PSF is fundamentally a non-linear function of the components of the electromagnetic field that manifests itself in the optical domain in the form of an energy distribution function. Mechanical dot gain was defined by Jackson (1990) as the overall growth in the shape of the printing element resulting from every step of printing process (separation of colours, production of printing plates, ink transfer, dye and substrate characteristics). Accordingly, mechanical dot gain is unique for every printing technique, since it is governed by the physical principles of generating the print. From this it is evident that every printing process results in mechanical dot gain, whereas every attempt of examining the print, results in optical dot gain, making them intertwined. For that reason, the value that is usually measured and modelled is the total dot gain using the Murray-Davies equation (Murray 1936).

One of the ways of quantifying a paper-ink system, and accordingly dot gain, is by applying the modulation transfer function, MTF. A lot of different, direct and indirect, methods of determining MTF are nowadays available, the most common one includes obtaining the reflectance profile of the printed edge, followed by its derivation and fitting the line spread function, LSF, and later applying a Fast Fourier Transform, FFT, to express it in the frequency domain. Previous examinations (Modrić et al. 2014) done with this method, as well as with Monte Carlo method of calculating subsurface scattering, showed an asymmetric LSF for the paper-ink system, more precisely, its one wing was better approximated by Lorentz function, while at the same time its other wing was better described with Gauss function, which indicates the existence of the above-mentioned mechanisms. Further research (Itrić et al. 2017) that included a new experimental method for determining the optical component in the PSF of a paper substrate by studying the interaction of collimated light and paper revealed that the optical component of the paper substrate PSF is symmetric and satisfactorily approximated with a Lorentzian function. With that in mind, we propose more accurate edge profiling as the key 
step for the future detailed characterization of the paperink system. Of course, the attempt of edge approximation is not a novelty in itself. Tzannes and Mooney (1995) have utilized curve fitting with several different analytical function models for finding the right fit of the edge spread function, ESF, such as Cauchy, Gumbel, hyperbolic, logistic and Gauss functions (analytical function models for the ESF fitting are called by their original names applied for the LSF fitting for simplicity reasons), which were later tested and compared by Li et al. (2009) who found that a logistic function gives the best result. Another function that intuitively comes to mind, primarily due to its origin (convolution of the Gaussian and Lorentzian), is Voigt's function as an appropriate solution for the LSF. Unfortunately, Voigt's function is symmetrical, whereas our profile is asymmetric which is why it was necessary to find a different solution, which appeared in the form of the bidose function.

It is well known fact that during the differentiation, generated noise is even greater than the noise in measured reflection profile of the edge, which makes edge fitting a suitable way of avoiding amplifying the noise during the derivative computation, which enables a more reliable prediction of the LSF and takes into account its asymmetry.

\section{Bi-logistic growth function}

The bi-logistic model was suggested in 1994 (Meyer 1994) to model systems that contain complex growth processes not adequately described by the simple logistic model, for occurrences where dual processes appear. Later on, in order to simulate the growth and diffusion processes with more than two sub-processes, the bi-logistic model was generalized to a component logistic model where growth is the sum of $n$ simple logistics.

The logistic growth assumes that the systems grow exponentially until an upper limit or carrying capacity inherent in the system is approached, at which point the growth rate slows and eventually saturates, while producing the characteristic S-shape curve (Stone 1993). In the case like ours, dealing with two logistic growth pulses, it is reasonable to split the data set in two and model each of them with separate logistic functions. The difficulties arise in defining where the first phase ends and the second one begins. Bi-logistic curves can be characterized into four types, the first one, sequential, describes two logistic growth pulses that do not overlap, in other words, the second phase does not start growing until the first is almost finished. The second type, superposed, involves the beginning of the second phase when the first phase has reached
$50 \%$. This model is appropriate for processes of similar nature. The remaining two models, converging and diverging, show asymmetric behaviour. The converging model shows a growth process where the first pulse of growth is joined by a second, faster one, so the both of them culminate about the same time. The last case, diverging bilogistic curve, includes two logistic growth processes that start at the same time but grow with different rates.

Our approach attempts to present dot gain as a system that experiences two different phases of growth, that either overlap or sequel each other, whose characteristics can be identified by analyzing the edge spread function. The first phase, mechanical dot gain, occurs during the reproduction and it is characterized with its own carrying capacity; a second phase, optical dot gain, has a different carrying capacity that superimposes on the first phase.

\section{Materials and methods}

In image analysis, edges can be considered as areas where the image pixel grey level values display a sharp variation. Certainly, the importance of edge stands to the fore due to its relevance in forming lines, curves and contours, since they represent outlines of solid objects. Intensity variations due to image noise are among the most significant challenges of edge detection. Clearly related is the other major issue of edge detection connected with the appropriate level of smoothening. Namely, higher smoothening has a desirable effect on noise reduction and other irregularities which makes future data processing easier. However, if used, it can result in the false position of the edge which implies that lower smoothening has a positive outcome in the resulting signal to noise ratio.

In this research, ESF will be approximated with a bidose function defined in Origin 9.5 as follows

$$
f=a_{1}+\left(a_{2}-a_{1}\right) \cdot\left[\frac{p}{1+10^{\left(b_{1}-x\right) h_{1}}}+\frac{1-p}{1+10^{\left(b_{2}-x\right) h_{2}}}\right]
$$

where $a_{1}$ and $a_{2}$ show minimum and maximum value, $b_{1}, b_{2}$ denote midpoint potency parameters (inflection points), $h_{1}$ and $h_{2}$ are the slopes of the two individual phases and $\mathrm{p}$ is the fraction of the phase that gives the larger contribution to the curve.

The research includes generating and analyzing monochromatic step samples (schematic is given in Figure 1). Five different computer to print (ctp) systems were used in the study, four of which are electrophotography devices (three with powder toner (E1, E2, E3) and one with liquid toner (E4)), while the remaining one is based 

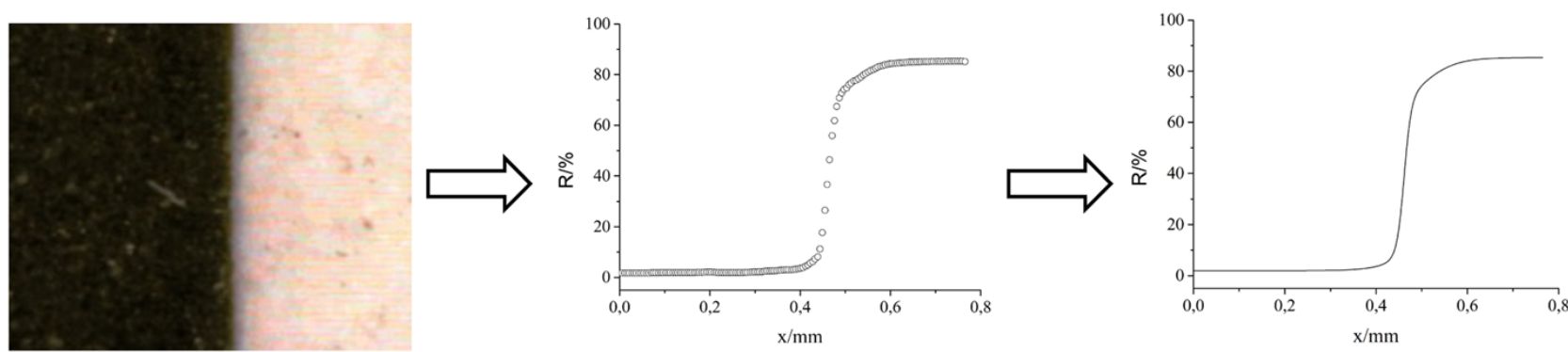

Figure 1: Process of obtaining edge spread function from printed edge profile, $R(x)$ (from left to right: picture of the edge, measured and smoothed reflection profile, fitted edge profile).

on direct imaging (DI), which means that the printing form is created in the device itself. Of course, the choice of paper also greatly determines the shape of the ESF; in this regard, three types of most commonly used papers in ctp technology were selected. Some general paper characteristics; weight (w), opacity (ISO 2471), whiteness (ISO 2470), roughness according to Bendtsen (ISO 8791/2) and crystallinity index-Cr.I. (ratio of absorbance peaks of Fourier Transform Infrared, FT-IR, spectra of plain paper at $1493 \mathrm{~cm}^{-1}$ and $893 \mathrm{~cm}^{-1}$ ) were determined. The crystallinity index was added since our previous research (Itrić et al. 2017) suggested the correlation of the ratio of mechanical/chemical pulp in the paper and the amount of scattering of light.

Quantitative analysis of prints was performed using Personal IAS Imaging Device (Quality Engineering Associates Inc). The validity of using the image analyzer is discussed in a large number of papers, among others in (Briggs and Tse 2005) and it will not be dealt with further in this paper. As far as PIAS itself, its component parts consist of a digital microscope (resolution $5 \mu \mathrm{m}$ and aperture $\left.(2.54 \times 2.54) \mathrm{mm}^{2}\right)$, and a CCD camera. It operates according to ISO/IEC 13660:2001 standards for measurement of image quality attributes for hardcopy output (binary monochrome text and graphic images). The software uses the system calibration curves and data from the internal reference target to convert the image to calibrated reflectance values (Briggs and Tse 2005). The illuminance of the measuring surface is diffuse in the visible area of the electromagnetic spectrum, while the measurement geometry is $45^{\circ} / 0^{\circ}$. Every process of image acquisition automatically implies generation of noise complicating further processing and identification of the results. Since we will be examining printed edge patterns, three separate noise sources come to question; paper granularity, non-uniformity of ink on paper and the measuring system noise. Due to this, reflectance profiles had to be smoothed wherein percentile filtering was chosen. Median filtering ( $50 \%$ percentile filtering) in which the value of the signal at every single point was replaced with the median value of the specific number of points from their neighbourhood (in our case 20 points were experimentally established) was chosen since the measured reflectance profiles contain localized spikes. The resulting reflection profiles were then fitted with the bidose function in Origin 9.5. The applied software uses the Levenberg-Marquardt method of nonlinear least-squares regression for estimating the parameters of logistic curves.

Printing direction also affects the appearance of the final edge profile since paper properties differ depending on the direction of formation of the sheet on the papermaking machine. The machine direction is also called the grain direction, whereas the direction perpendicular to the machine direction is known as the cross-machine direction, or cross-grain direction. Exactly due to these reasons, measurements are included that encompass both situations, machine direction and cross direction printing.

\section{Results}

Measured paper characteristics are given in Table 1.

A graph depicting measured and fitted edge for one of the systems is shown in Figure 2. At the same time, LSF is also presented to confirm the impact of a better-defined edge on the amount of noise in the derivative.

If we consider the peak of the LSF as the real position of the edge, a definite trend in the fitting data can be seen.

Table 1: Basic characteristics of the papers used in the study.

\begin{tabular}{lrrrrr}
\hline Paper & $\begin{array}{r}\text { Weight } \\
\mathbf{g} \cdot \mathbf{m}^{-2}\end{array}$ & $\begin{array}{r}\text { Opacity } \\
\%\end{array}$ & $\begin{array}{r}\text { White } \\
\%\end{array}$ & $\begin{array}{r}\text { Rough } \\
\mathbf{m l}^{\prime} \cdot \mathbf{m i n}^{-1}\end{array}$ & Cr.I. \\
\hline P1 & 115 & 91 & 112 & 70 & 0.67 \\
P2 & 80 & 98 & 110 & $75-175$ & 0.59 \\
P3 & 120 & 96 & 110 & 265 & 0.81 \\
\hline
\end{tabular}




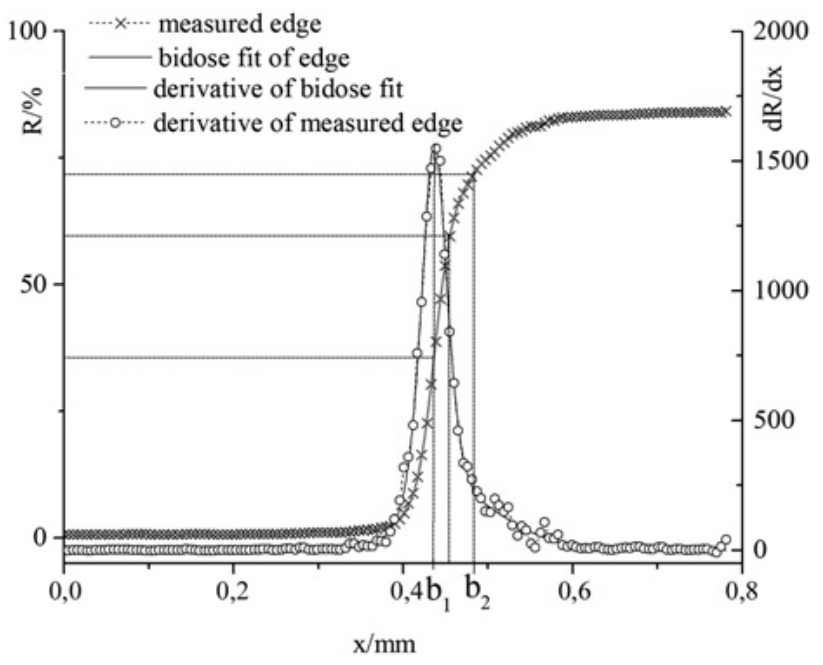

Figure 2: Measured and fitted ESF for E1-P1 sample together with the derivatives of the measured edge and its bidose fit.

The inflection point of the first phase, $b_{1}$, corresponds with the peak of the LSF in all of the samples. Further on, the area between two inflection points, $b_{1}$ and $b_{2}$, can be understood as the interval in which mechanical and optical dot gain overlap, while the values before the first, and following the second inflection point can be assigned exclusively to mechanical and optical dot gain.

It is clear from the obtained data (Table 2) that all of the samples based on dry-toner electrophotography, regardless of the fibrous media used for printing, show almost the same fraction of the curve comprising the more pronounced phase (70-75\%), and we believe that this phase corresponds to mechanical dot gain. The remaining part corresponds to optical dot gain.

Powder toner generates a Gaussian profile of the edge due to various reasons. One possibility lies within the imaging process in the toner surface, which leads to lower viscosity compared to liquid toner. Secondly, the transfer mechanism is less well-defined spatially with powder toner, and finally the levelling of the dry toner on melting is greater than the surface energy-driven spread of the liquid toner, which manifests in the edge structure. In com- parison to powder toner electrophotography, liquid toner electrophotography exhibit a smaller fraction of the curve comprising the mechanical dot gain, which is consistent with previous knowledge about the quality of prints with powder vs liquid toner. Namely, dry-toner electrophotography is strongly dependent on the electrostatic properties of the substrate, meaning that even the smallest change in relative humidity may result in considerable changes in the print quality. Regarding the influence of machine / cross direction printing, it is evident that printing in cross direction results in higher noise (Figure 3), indicating even more pronounced noise in the LSF, which is one of the main reasons traditional methods of obtaining LSF were not adequate for examination of cross direction prints.

Likewise, printing direction heavily influences the ratio of mechanical/optical dot gain implying, that the higher ratio of mechanical dot gain is better for final product quality.

If we compare the values for direct imaging with those for electrophotography, it can be concluded that higher the value of $p$ indicates higher quality of the final product.

\section{Discussion}

It is interesting to note that previous research by (Modric et al. 2009) indicated that the fraction of light that penetrates the substrate corresponds to $30 \%$, which is in accordance with the current data obtained in this study. We see that the first phase is obviously faster than the second, indicated with the almost four times higher slope in comparison to the slope of the second phase.

In the previous work done by our group, the reflectance profile of a screen element was obtained as the convolution of the LSF with a modeled reflectance profile of that element. It was found that the LSF can be better approximated by means of the Lorentzian function when compared to the Gaussian profile that was used in the past research (Modrić et al. 2014). For comparison, the LSF modelled with a Gauss and Lorentz function, as well as the

Table 2: Parameters of the fitted bidose functions for all of the samples.

\begin{tabular}{lrrrrrrrr}
\hline Sample & $\mathbf{r}^{\mathbf{2}}$ & $\mathrm{a}_{\mathbf{1}} / \%$ & $\mathrm{a}_{\mathbf{2}} / \%$ & $\mathbf{b}_{\mathbf{1}} / \mathbf{m m}$ & $\mathbf{b}_{\mathbf{2}} / \mathbf{m m}$ & $\mathbf{h}_{\mathbf{1}} / \mathrm{mm}^{-\mathbf{1}}$ & $\mathbf{h}_{\mathbf{2}} / \mathbf{m m}^{-1}$ & $\mathbf{p}$ \\
\hline E1-P1 & 0.99998 & 0.726 & 83.858 & 0.4366 & 0.4778 & 42.795 & 12.373 & 0.7021 \\
E2-P2 & 0.99968 & 4.594 & 84.012 & 0.4034 & 0.4664 & 52.563 & 12.726 & 0.7412 \\
E3-P3 & 0.99994 & 4.431 & 83.646 & 0.3402 & 0.3869 & 45.671 & 10.418 & 0.7525 \\
DI-P3 & 0.99984 & 9.294 & 83.428 & 0.3833 & 0.4282 & 52.168 & 12.455 & 0.7736 \\
E4-P1H & 0.99987 & 8.167 & 75.361 & 0.5037 & 0.5292 & 39.980 & 11.731 & 0.6312 \\
E4-P1V & 0.99979 & 4.912 & 71.483 & 0.5162 & 0.5226 & 53.395 & 11.341 & 0.5171 \\
\hline
\end{tabular}



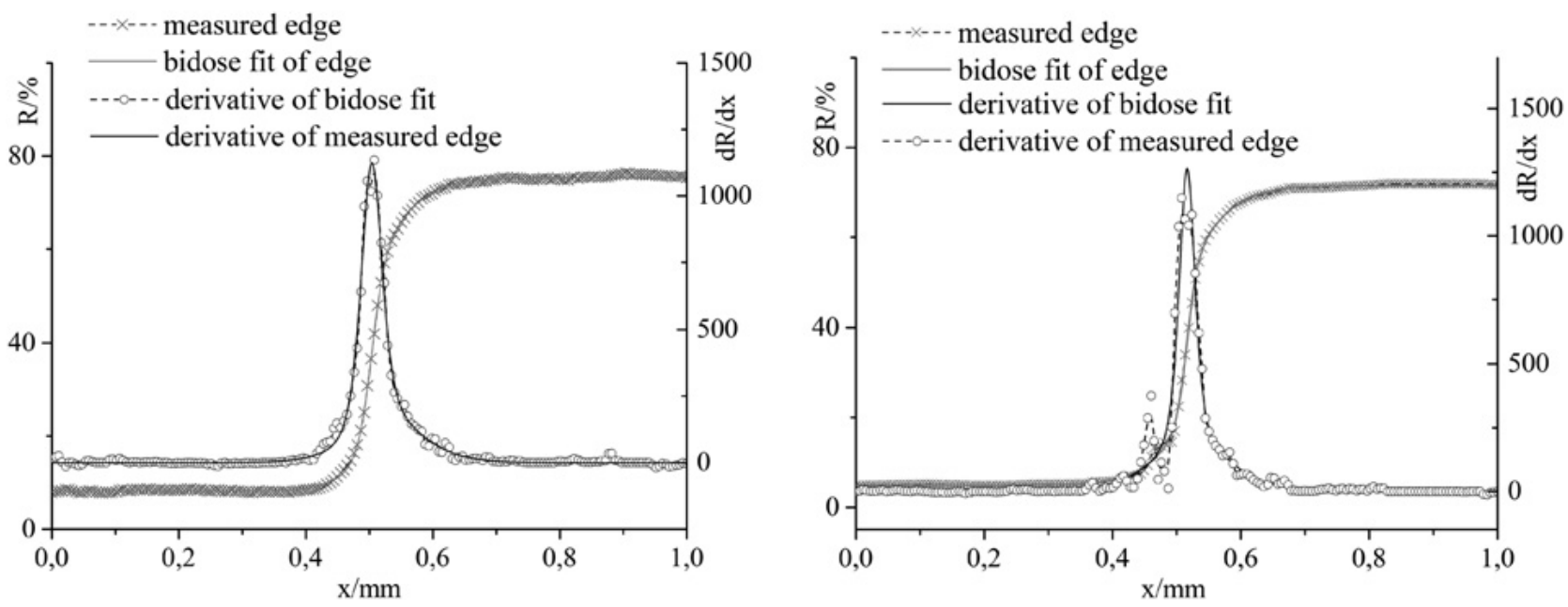

Figure 3: a) Measured and fitted ESF for E4-P1H sample together with the derivatives of the measured edge and it's bidose fit; b) Measured and fitted ESF for E4-P1V sample together with the derivatives of the measured edge and it's bidose fit.

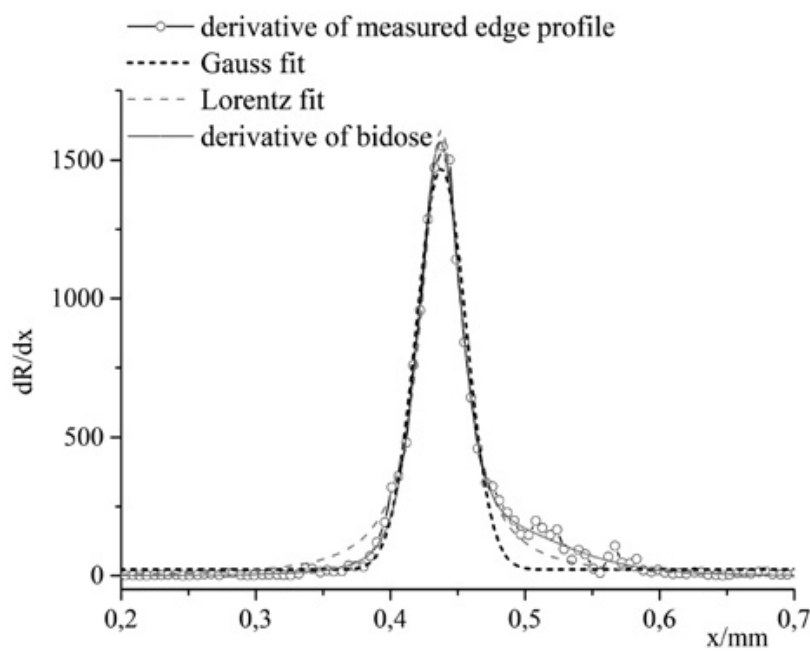

Figure 4: LSF of E1-P1 approximated with Gauss, Lorentz and derivative of bidose functions.

LSF modelled as the derivative of a fitted bidose function is given in Figure 4.

Clearly, the best fit of the LSF is shown in Figure 4 by the derivative of the bidose function.

The value of the dot gain itself does not present much of a problem for print quality as the edge structure (definition/shape). Therefore, using the slope of a bi-logistic growth function approximation directly addresses quality of the press, considering that these profiles are averaged over the length of the line. Accordingly, the whole problem can be seen as a complex probability of the reflection of the photon from the surface of the imprint depending on two completely different physical processes. Our approach is not limited to studying dot gain, rather it can be easily applied to image analysis in various scientific fields (e. g. medicine, astronomy, materials science, robotics) if the matching conditions are met, i.e. first of all, there are two sub processes that define them (for example a combination of two functions in optics mapping-Gaussian and Airy), and secondly, the LSF should be asymmetric. Given the extremely high correlation coefficient with the bidose function, we consider this description to be a good approximation of the analytical function by which we describe the shape of the edge.

\section{Conclusions}

Dot gain could be controlled precisely throughout the printing process if its parameters are well known. Our approach, based on the more precise fit of the edge spread function with a bidose function shows a more than satisfactory coefficient of correlation $\left(r^{2}>0.999\right)$ for all of the tested samples. At the same time, it has been found that in all prints generated with powdered toner electrophotography, as well as direct imaging, the degree of the curve comprising the more pronounced phase (mechanical dot gain) is greater than $70 \%$, while this number is slightly lower for liquid toner electrophotography.

So far, it has been shown that the optical component is better described with a Lorentz function, while the mechanical contribution is better described by a Gauss function. The overall probability that the photon leaves the surface of the paper is a mixture of the two contributions. Until now, all accessible literature dealt with cases where both functions are of the same type (e.g. Gaussian), and 
the result of mixing is a symmetric function. In the present case, this is not so because the resultant function is asymmetric, indicating the need for a better theoretical description, and we have shown that the derivative of the bidose function does not generate simultaneously both Lorentz and Gauss contributions.

Regarding different directions of printing in relation to the direction of paper formation, we can definitely conclude on a phenomenological level that a slope of the more pronounced phase (mechanical dot gain) is highly influenced by the direction of print, while the second phase (optical dot gain) remains almost the same.

It is clear that much additional work will be required before a complete understanding of the phenomena can be reached, but this approach based on image analysis allows us to identify the domain of the impact of a particular contribution, which has not been done so far.

Conflict of interest: The authors declare no conflict of interest.

\section{References}

Briggs, J.C., Tse, M.-K. (2005) Objective Print Quality Analysis and The Portable Personal IAS $®$ Image Analysis System. NIHON GAZO GAKKAISHI (Journal Imaging Soc. Japan) 44(6):505-513.
Itrić, K., Modrić, D., Hladnik, A. (2017) A novel method for determining the optical component of the paper substrate point spread function. Optik (Stuttg) 140.

Jackson, L.L. (1990) Optical dot gain and the off-press color proof. Graphic Arts Technical Foundation.

Li, T., Feng, H., Xu, Z., Li, X., Cen, Z., Li, Q. (2009) Comparison of different analytical edge spread function models for MTF calculation using curve-fitting. 7498, 74981H.

Meyer, P. (1994) Bi-logistic growth. Technol. Forecast. Soc. Change 47(1):89-102.

Modrić, D., Bolanča, S., Beuc, R. (2009) Monte Carlo modeling of light scattering in paper. J. Imaging Sci. Technol. 53(2):20201.

Modrić, D., Petric Maretić, K., Hladnik, A. (2014) Determination of point-spread function of paper substrate based on light-scattering simulation. Appl. Opt. 53(33):7854.

Murray, A. (1936) Monochrome reproduction in photoengraving. J. Franklin Inst. 221(6):721-744.

Rogers, G.L. (1997) Optical dot gain in a halftone print. J. Imaging Sci. Technol. 41(6):643-656.

Rogers, G.L. (1998) Effect of light scatter on halftone color. J. Opt. Soc. Am. A 15(7):1813-1821.

Stone, R. (1993) Sigmoids. Bull. Appl. Stat. 7:5-11.

Tzannes, A.P., Mooney, J.M. (1995) Measurement of the modulation transfer function of infrared cameras. Opt. Eng. 34(6):1808-1818. 